\title{
Cholesterol in health and disease
}

\author{
Ira Tabas \\ Department of Medicine, and Department of Anatomy and Cell Biology, Columbia University, New York, New York, USA \\ J. Clin. Invest. 110:583-590 (2002). doi:10.1172/JCI200216381.
}

Imagine if an excess amount of a critical, life-sustaining molecule like ATP were, by a perverse series of events involving the lifestyle of modern humans, causally related to a major human disease. The thought of "ATP" being synonymous with poor health and poor living in the minds of the lay public and press, even of health care providers, would be difficult for any selfrespecting scientist to accept. So one might view the biomedical history of cholesterol - indeed, this history might be seen as even stranger than the hypothetical ATP scenario, given that evolution has devoted close to 100 genes to the synthesis, transport, metabolism, and regulation of cholesterol. This structurally fascinating lipid is utterly essential to the proper functioning of cells and organisms. Cholesterol, cholesterol metabolites, and immediate biosynthetic precursors of cholesterol play essential roles in cellular membrane physiology, dietary nutrient absorption, reproductive biology, stress responses, salt and water balance, and calcium metabolism. Indeed, many of the articles in this Perspective series are devoted to the normal physiology of cholesterol. Still, there is little doubt that the disease process responsible for the leading cause of death in the industrialized world - atherosclerosis - is a disorder in which an excess of cholesterol is a major culprit. How did evolution come up with a molecule that is critical for so many aspects of normal physiology, and what went "wrong"?

\section{The physiology of cholesterol}

Cholesterol and cellular membranes: an evolutionary perspective. As organisms became more complex, cells required membranes that would provide the proper conformational environment for a wide variety of integral membrane proteins, such as channels, transporters, and enzymes. Moreover, the cells of these advanced organisms required sophisticated signaling machinery, and this machinery would have to be organized as multiprotein complexes in focal, nonhomogenous areas of cellular membranes. Put simply, these requirements were met by focally increasing the "stiffness" or viscosity of phospholipid bilayers. In theory, this goal could be achieved

Address correspondence to: Ira Tabas, Department of Medicine, Columbia University, 630 West 168th Street, New York, New York 10032, USA. Phone: (212) 305-9430; Fax: (212) 305-4834;

E-mail: iat1@columbia.edu.

Conflict of interest: No conflict of interest has been declared. by increasing the degree of saturation of the fatty acyl moieties of membrane phospholipids. However, unsaturated fatty acids in these phospholipids, particularly in the $s n-2$ position, are needed for a wide variety of cellular signaling functions, and so an exogenous "stiffening factor" was needed. This factor would have to be able to pack tightly with the long saturated and unsaturated fatty acyl chains of membrane phospholipids through van der Waals interactions. This would require a long, flat, and properly shaped hydrophobic molecule, accompanied by additional features (see below) to help further stabilize this interaction.

To meet these needs, nature began with molecules, probably originating from prebiotic anaerobic times, that were formed by the sequential condensation of the two-carbon acetate molecule. Prokaryotes evolved enzymes to synthesize more complex linear molecules from these precursors (e.g., carotenoids), which were able to meet the membrane-organizing requirements of most of these primitive organisms. These primitive molecules, however, lacked the proper shape, rigidity, and amphipathic nature to properly organize the membranes of more advanced organisms and, interestingly, two species of bacteria (see below) (1). At some point in the evolution of the earliest eukaryotes or their nearest Archaeal relatives, the largest of these linear molecules was cyclized to form steroids, planar structures containing four rings (2). This shape change conferred rigidity and altered the molecule's ability to pack within the fatty acyl tails of neighboring phospholipids. Additional features were added that helped stabilize these lateral interactions, including the introduction of a C-C double bond (3) and of an -OH group, which converted the steroid to a sterol; the precise role of the latter modification is controversial (1).

These molecular transformations correspond to the initial conversion of acetate into acyclic, apolar squalene, followed evolutionarily by the oxygenation and cyclization of squalene to lanosterol (Figure 1). Lanosterol is then converted to cholesterol by oxidative demethylation. As referred to above, two species of bacteria, Methylococcus capsulatus and Mycoplasma species, require sterols for growth; while Mycoplasma obtains these sterols from the cells of higher organisms, $M$. capsulatus actually synthesizes sterols (1). In both cases, however, the structural requirements of these sterols are more lax than those described above for eukaryotes (1). Interestingly, the cyclization of squalene and the conversion of lanosterol 


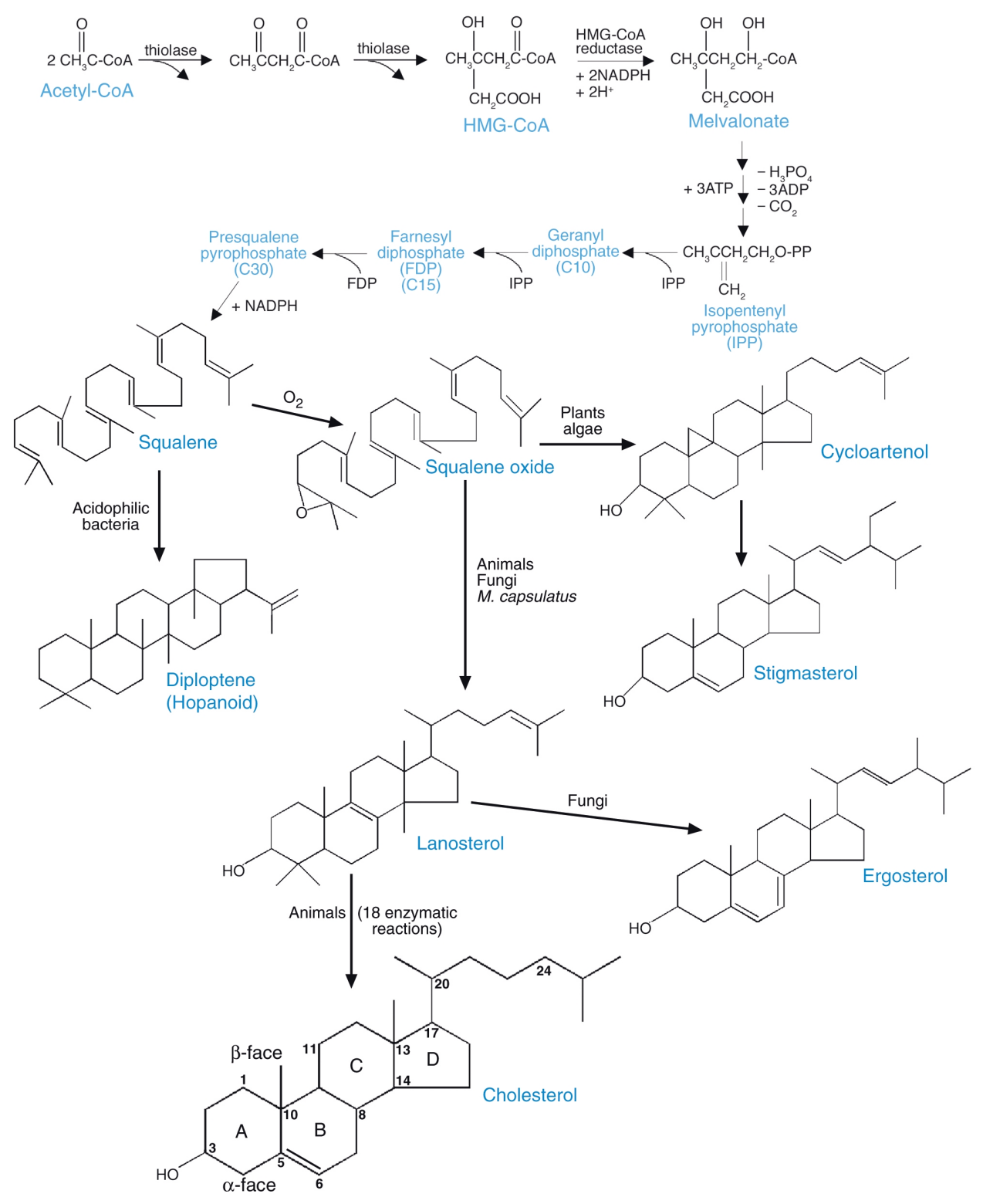

Figure 1

Biosynthesis of steroids in various species. Adapted from ref. 43 with permission of the editors and publisher.

to cholesterol require molecular oxygen and could therefore occur only after the evolution of aerobic cells. However, in a fascinating "evolutionary preview" of some of these features, acidophilic bacteria evolved enzymes to anaerobically synthesize cyclic membrane-organizing lipids called hopanoids from squalene (Figure 1). Haines has speculated that hopanoids fill a unique role in acidophile membrane structure, namely, the prevention of inward leakage of protons (4).

The structural requirements outlined above demand precision. For example, a molecule almost identical to cholesterol but with a $3 \alpha$-hydroxyl group instead of a $3 \beta$-hydroxyl group ("epicholesterol") cannot function properly in biological membranes (5). Moreover, the conversion of lanosterol to cholesterol involves a complex series of 18 enzymatic reactions, even though lanosterol is already a cyclic $3 \beta$ alcohol. In fact, lanosterol and other methylated derivatives cannot substitute for cholesterol in mammalian cell mutants that are auxotrophic for sterols (6). Why? The three methyl groups that are removed from lanosterol to form cholesterol are all on the so-called $\alpha$-face of the molecule and thus protrude from this otherwise flat surface (Figure 2a). This is particularly true of the axial $14 \alpha$-methyl group of lanosterol. Bloch and others have suggested that the removal of these three methyl groups allows proper fitting of the ring structure to the fatty acyl chains of membrane phospholipids, particularly those that are saturated $(1,7)$ (Figure $2 \mathrm{~b}$ ). According to this model, cholesterol optimally binds natural membrane phospholipids, which usually contain a saturated fatty acid at $s n-1$ and an unsaturated fatty acid at $s n-2$. In par- 
ticular, its flat $\alpha$-face is ideally suited to interact with the $s n$ - 1 saturated fatty acyl group, whereas the methylated $\beta$-face is optimal for interaction with the more flexible unsaturated fatty acyl chains in the $s n-2$ position of an adjacent phospholipid molecule. The $\alpha$-face of cholesterol, but not lanosterol, has the additional feature of a C-C double bond, which further enhances interaction with neighboring phospholipids (3). In this context, Mouritsen and colleagues (8) have used deuterium nuclear magnetic resonance spectroscopy on model membranes to demonstrate the advantage of cholesterol over lanosterol in the formation of liquidordered membrane domains, or "rafts" (see below).

Finally, the side chain of cholesterol is saturated and relatively unsubstituted with extra methyl groups, resulting in a hydrophobic structure that is usually linear and aligned with the plane of the steroid nucleus (Figure 2a). This design allows optimal van der Waals interactions with phospholipid fatty acyl groups while avoiding disruption of the bilayer $(1,7)$. Interestingly, plants evolved a different series of enzymatic reactions in which squalene is converted to cycloartenol instead of lanosterol, followed by further modifications that result in the synthesis of phytosterols (Figure 1). Phytosterols are characterized by double bonds and additional methyl and/or ethyl substitutions in the side chain. These bulky side chains, which are often aligned out of the plane of the steroid nucleus, alter critical properties of the membranes of higher animal cells (9). Indeed, higher animals have evolved specific, energyrequiring molecules to excrete such sterols following intestinal absorption (10). Plant cell plasma membranes, however, encounter a much higher proton gradient than most animal cell plasma membranes, and the bulky side chains of phytosterols appear to be optimally designed to prevent proton leaks, thus saving the cells a great expenditure of metabolic energy (4).

The result of the molecular evolution of cholesterol is an exquisitely designed molecule that can influence many important properties of the vertebrate plasma membrane. In addition to generally affecting membrane permeability and integral membrane protein function, cholesterol-induced membrane packing in lateral microdomains, or rafts, of the plasma membrane can provide a scaffold for a variety of membraneassociated signaling proteins, as reviewed by Simons and Ehehalt (11) in this Perspective series. As such, the role of cholesterol in the proper functioning of oncogenic $G$ proteins (12), proteases of amyloid precursor protein (13), and signaling proteins critical for sperm activation (Travis and Kopf [14], this series) extends the biomedical implications of cholesterol from cancer to Alzheimer disease to reproductive biology. Moreover, recent work has revealed a critical role for cholesterol in glial cell-mediated synaptogenesis, perhaps by directly affecting the structures of synaptic vesicles and postsynaptic membranes or by activating a neuronal signaling pathway (15). Cholesterol can also influence the localization of a protein important in vertebrate development, Sonic hedgehog, by covalently attaching to the protein itself (Jeong and McMa- hon [16], this series). The formation of cholesterol-rich microdomains in the plasma membrane is also critical for the budding of clathrin-coated pits (17), a key step in receptor-mediated endocytosis. The influence of cholesterol on normal cellular physiology is clearly demonstrated by what happens when cells are experimentally depleted of cholesterol or when, by accidents of nature, mutations prevent the proper synthesis or trafficking of cholesterol. The specific effects of cholesterol biosynthetic mutations are covered by Porter (18) in this series, and the Perspectives by Maxfield and Wüstner (19) and by Jefcoate (20) will discuss mechanisms of and mutations in specific aspects of intracellular cholesterol trafficking.

The critical membrane-organizing functions of cholesterol justify the evolution of an elaborate feedback regulatory system. As described by Horton, Goldstein, and Brown in a recent Spotlight in the JCI (21), sterol starvation induces the translocation of SREBP-SCAP complex to the Golgi apparatus, where two proteolytic cleavages generate a transcriptional activator that induces genes controlling both cholesterol biosynthesis and exogenous cholesterol uptake. Interestingly, insect cells have all of the components of this regulatory system, yet they do not synthesize cholesterol, and their ability to process SREBP is not affected by exogenous sterols. Rather, the system is used in phosphatidylethanolamine-mediated feedback regulation of the biosynthesis of fatty acids and phospholipids, which critically affect membrane structure in these cells (22). Because phatidylethanolamine and cholesterol share the ability to alter membrane structure and thus to alter SCAP conformation and SREBP processing (22), vertebrate evolution appears to have usurped a pre-existing system that was devoted to membrane
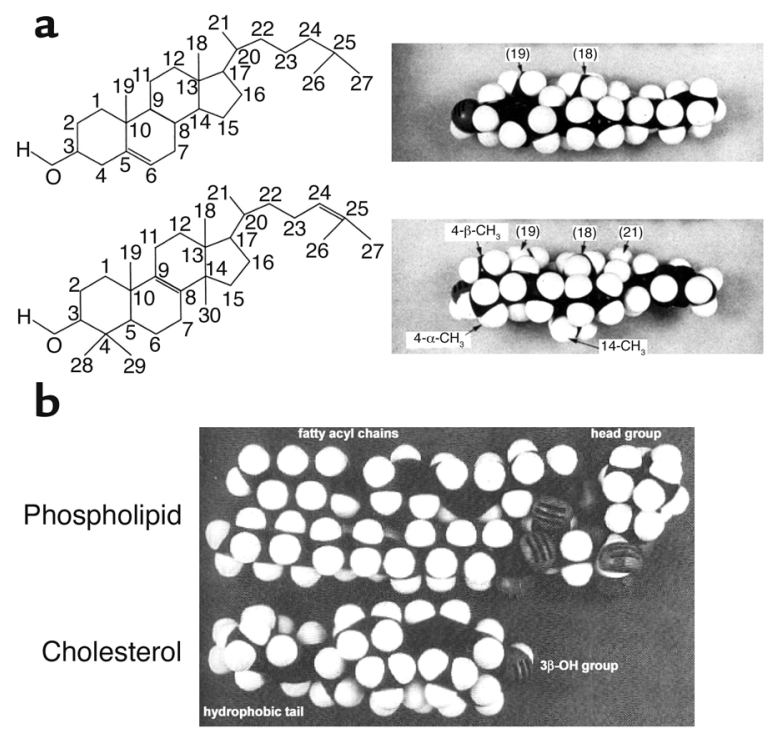

Figure 2

(a) Structural and space-filling models of cholesterol and lanosterol. Reproduced from ref. 8 with permission of the author and journal. (b) Space-filling model of cholesterol interacting with a phospholipid molecule. Reproduced from ref. 7 with permission of the author and journal. 


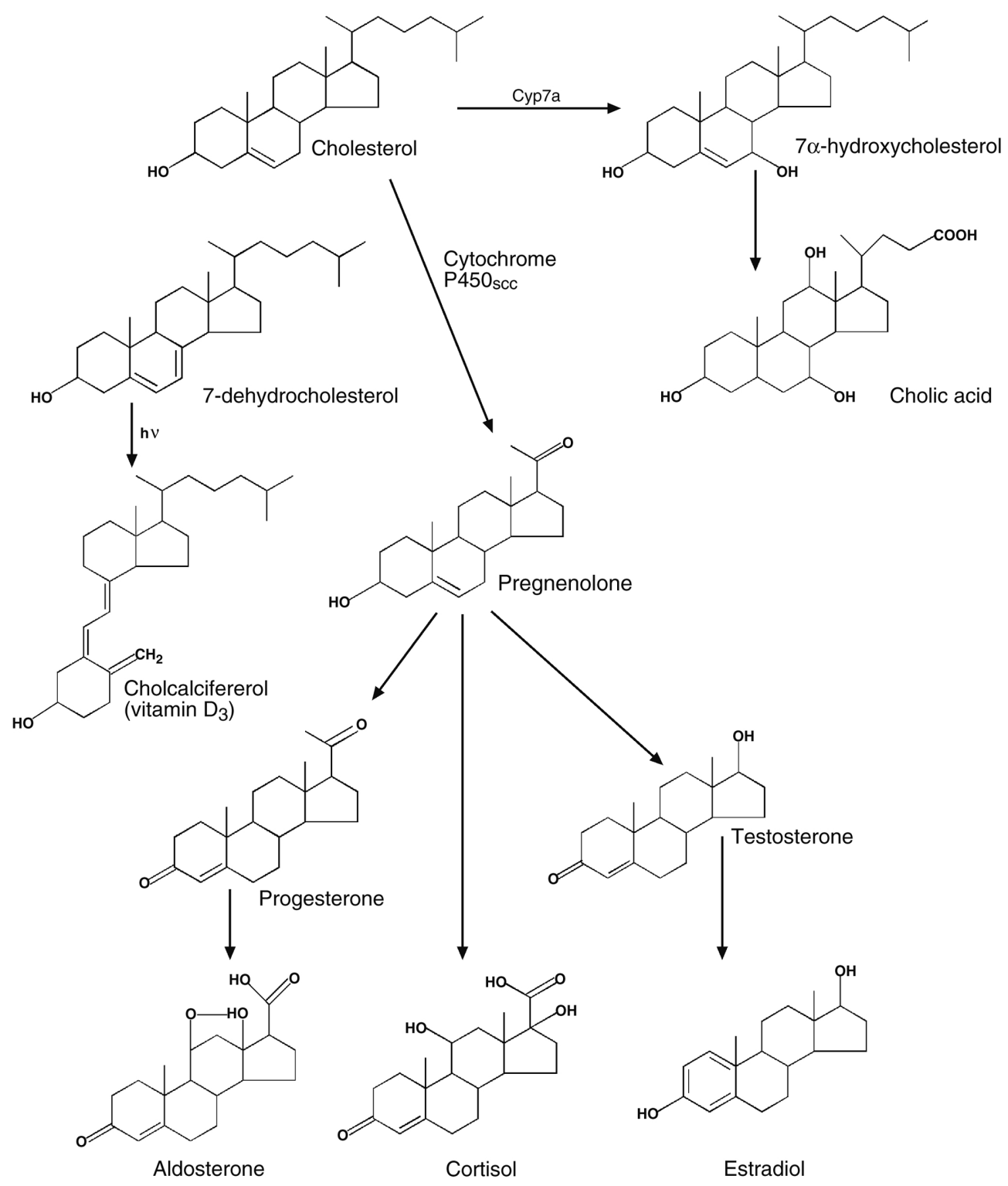

Figure 3

Biosynthesis of cholesterol-derived hormones. Adapted from ref. 43 with permission of the editors and publisher.

lipid homeostasis even before cholesterol emerged as the key molecular organizer of cell membranes.

Functions of cholesterol metabolites and immediate biosynthetic precursors of cholesterol. As if the membrane effects of cholesterol were not enough, nature has used the cholesterol backbone as a precursor for a variety of biologically active molecules (Figures 3 and 4). In one pathway, at least 14 different liver enzymes add polarity to the ring structure and side chain of cholesterol to create ideal micellar solubilization agents for the absorption of dietary fats and fat-soluble vitamins (23). Specifically, these solubilizing agents, or bile acids, have a carboxyl group on the normally apolar side chain of cholesterol and one to two extra hydroxyl groups on the ring structure itself (Figure 3; see Björkhem [24], this series). Importantly, bile acids (and thus cholesterol) are conserved during the process of lipid absorp- tion through the enterohepatic circulation. Thus, by facilitating cholesterol absorption, bile acids ensure adequate supplies of total body cholesterol when this critical lipid is in short supply. This model is consistent with the finding that the rate-limiting enzyme of bile acid synthesis, cholesterol $7 \alpha$-hydroxylase, is downregulated by excess hepatic cholesterol in primates (25). However, pharmacologic interruption of the enterohepatic circulation of bile acids leads to increased conversion of hepatic cholesterol into bile acids, thus converting the bile acid pathway into one that can actually help eliminate excess body cholesterol (23).

In an interesting twist of evolution, the liver of the spermiating adult male sea lamprey, an ancestral jawless fish, synthesizes a sulfated bile acid (26) (Figure 4). However, these fish have neither bile ducts nor gall bladders, and they do not feed while spermiating. Rather, 
<smiles>CC(=O)C1CCC2C3CCC4CC(O)CCC4(C)C3CCC12C</smiles>

$3 \alpha, 5 \alpha$-tetrahydroprogesterone (neurosteroid)<smiles>CC(C(O)CCC(C)(C)O)C1CCC2(O)C3=CC(=O)C4CC(O)C(O)CC4(C)C3CCC12C</smiles>

Ecdysone
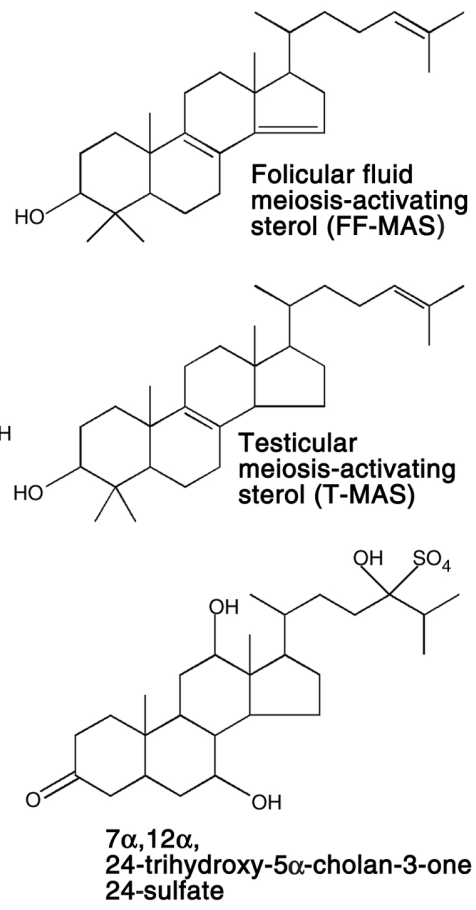

24-trihydroxy-5 $\alpha$-cholan-3-one

Figure 4

Structure of sterol-related molecules with unusual biological functions. See text for details.

the bile acid is transported to the gills, where specialized glands secrete the substance into surrounding waters. There, it functions as an ideal long-distance pheromone to attract females to nests built by the males (26).

Perspectives by Björkhem (24) and by Tall and colleagues (27) in this series discuss additional cholesterol side-chain oxidation reactions that may have important effects in cholesterol metabolism in a variety of tissues. Work in vitro has demonstrated that cholesterol modified by the addition of one additional hydroxyl group on the side chain creates a molecule that is able to activate a nuclear hormone receptor called LXR (28). As discussed by Tall and colleagues, heterodimers of activated LXR and RXR transcriptionally induce a series of genes involved in the efflux, transport, and hepatic metabolism of "excess" cholesterol derived from peripheral cells. Thus, it is possible that specific side-chain hydroxylases play a critical role in so-called reverse cholesterol transport. Oxidation of the side chain to a hydroxyl or carboxylic acid group may also directly promote the efflux of sterols from cells by increasing their aqueous solubility (Björkhem, this series).

Another scenario involves the oxidative cleavage of the side chain of cholesterol to a C20 ketone moiety (Figure 3). This reaction is catalyzed by a specific cytochrome P450 enzyme in the mitochondria of steroidogenic cells (see Jefcoate [20], this series) and possibly brain cells. The resulting molecule, $\Delta-5$ pregnenolone, is a precursor for a large number of new molecules that have the proper shape to bind and activate nuclear hormone receptors involved in a variety of critical metabolic processes (29) (Figure 3). In certain cases, the $\mathrm{C} 20$ ketone moiety of pregnenolone is retained, and the $3 \beta$-hydroxyl group is oxidized to a ketone (e.g., progesterone, cortisol, and aldosterone) (Figure 3). In other cases, the side chain is oxidatively cleaved further to yield a C17 hydroxyl group (e.g., testosterone and estradiol), and in one of these molecules (estradiol) the A ring is reduced to a phenol (Figure 3). Different degrees of ring demethylations, oxidations, and reductions add further variety to the shape of the steroid nucleus and thus confer their specificity for individual hormone receptors (29).

In the classic pathway defined for steroid hormone signaling, the hormone-receptor complex enters the nucleus and transcriptionally activates genes critically involved in reproductive biology (e.g., progesterone, estradiol, and testosterone), the stress response (e.g., cortisol), intravascular salt and volume homeostasis (e.g., aldosterone), and possibly higher brain functions (e.g., sex hormones) (29). An evolutionary preview of this paradigm is found in insects, where exogenously derived sterols are oxidized to form
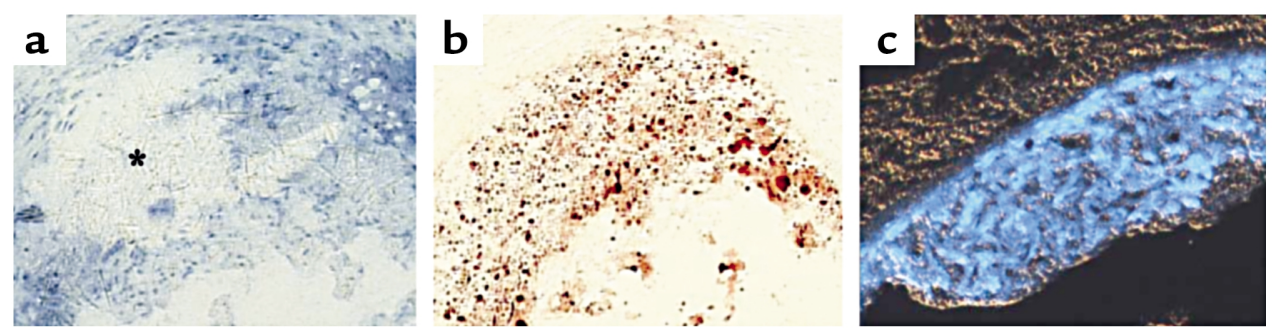

\section{Figure 5}

Cholesterol in atherosclerosis. The images show proximal aorta atherosclerotic lesions from the atherosclerosis-susceptible apoE knockout mice fed a cholesterol- and saturated fat-enriched diet for 25 weeks. ( $\mathbf{a}$ and $\mathbf{b}$ ) Adjacent sections from the same lesion stained with hematoxylin (a) or oil red $\mathrm{O}$ (b). The asterisk in a marks an area filled with cholesterol crystals. The bright red-orange staining in $\mathbf{b}$ shows areas rich in neutral lipids, most likely cholesteryl ester inclusions in macrophage foam cells and cholesteryl ester-rich emulsions in extracellular regions of the lesion. Areas of the lesion that stain poorly with oil red $\mathrm{O}$ are often rich in free (i.e., unesterified) cholesterol. (c) Fluorescence microscopy image of another atherosclerotic lesion stained with the fluorescent dye filipin (shown as blue in this image), which binds specifically to areas enriched in free cholesterol. The bright filipin staining in almost the entire diseased intima indicates that advanced atherosclerotic lesions in this model are very rich in free cholesterol. 
ecdysteroids (Figure 4), which control metamorphosis by interacting with nuclear hormone receptors (30). Interestingly, recent data have suggested that the activation of estrogen and androgen receptors by sex hormones can lead to nongenotropic effects in cells, such as attenuation of apoptosis (31). Similarly, certain neurosteroids can directly modulate the activity of GABA receptors in the brain (32) (Figure 4).

Finally, nature has also taken advantage of some of the immediate precursors of cholesterol to fulfill other critical functions. For example, 7-dehydrocholesterol in the skin is converted to cholecalciferol (vitamin D3) via cleavage of the $B$ ring by near ultraviolet irradiation (33) (Figure 3). A similar reaction occurs in plants, where ergosterol is converted to ergocalciferol (vitamin D2). Further hydroxylations at the $\mathrm{C} 25$ and $\mathrm{C} 1$ positions lead to the hormonally active molecule 1,25dihydroxy-vitamin $\mathrm{D}$, which interacts with a nuclear hormone receptor that critically affects body calcium metabolism (33). Likewise, lanosterol-derived molecules called meiosis-activating sterols are employed by mammals to activate resumption of meiosis in oocytes and spermatozoa (34) (Figure 4).

In summary, by oxidative cyclization and demethylation of an acetate-derived hydrophobic molecule, nature has created an amphipathic planar structure that, directly or through modifications, affects an incredible array of critical biological processes. These processes affect cellular membrane physiology, dietary nutrient absorption, reproductive biology, stress responses, salt and water balance, and calcium metabolism. Each of these processes is absolutely necessary for organisms to reach reproductive age. Thus, there has been strong evolutionary pressure to ensure that the body in general and individual cells in particular have an adequate supply of cholesterol. Indeed, the regulatory response of cells to sterol starvation, as mentioned above, is exquisitely designed for this purpose. These regulatory processes can also protect cells from moderate degrees of cholesterol excess. What nature did not plan for, however, was how to handle levels of cholesterol that exceed these limits.

\section{The pathophysiology of cholesterol}

Homo sapiens has many physical characteristics of a vegetarian organism (e.g., structure of teeth and intestines, sipping rather than lapping to drink, sweating rather than panting to moderate body temperature) and evolved with the high physical activity of a huntergatherer (35). As such, intestinal and hepatic lipid metabolism likely evolved to convert whatever dietary fats were available into a form, namely, triglyceriderich lipoproteins, that could supply energy to tissues. The limited amount of cholesterol in the hunter-gatherer's diet was not a problem, because most cells have a more than adequate capacity to make all of the cholesterol they need for membrane structural functions (36). Indeed, the way cells ensure proper levels of endogenous cholesterol synthesis when exogenous sources are low is part of the exquisite story of cellular regulation that was described recently in the JCI by
Horton, Goldstein, and Brown (21). Those cells that use cholesterol as a precursor to other molecules, such as steroidogenic cells (steroid hormones) and hepatocytes (bile acids), may need an exogenous supply under certain conditions, but this could easily be met by the internalization of the small amount of cholesterol left in the remnants of triglyceride-rich lipoproteins or in the LDL-like and HDL-like by-products of triglyceriderich lipoprotein metabolism.

Intruding on this serene picture of metabolic harmony was the development of the superior intellect of modern humans, which enabled our species to secure the higher energy content of animal-derived foods at a much lower cost to metabolic energy. For reasons that are still not clear, the ingestion of saturated fats often causes a dramatic rise in plasma cholesterol levels, including those in remnant lipoproteins and LDL. The increase in plasma cholesterol is further exacerbated by obesity, insulin resistance, and perturbations of glucose and fatty acid metabolism, all of which accompany sedentary lifestyles.

Even with these problems, the degree of plasma cholesterol elevation that occurs at or before reproductive age usually poses no immediate threat. For cells that internalize some of this excess cholesterol, there are multiple protective mechanisms, including downregulation of endogenous cholesterol biosynthesis and of LDL receptors, intracellular esterification of the excess cholesterol by acyl-coenzyme A:cholesterol acyltransferase, and efflux of cholesterol by multiple pathways (37). For tissues, the adverse reactions that occur in response to the accumulation of large amounts of extracellular cholesterol-rich lipoproteins take years to develop.

In post-reproductive life, however, the pressures of evolution have little impact, and the toll for elevated cholesterol is eventually paid. Clearly, the highest toll is associated with atherosclerotic vascular disease, although other consequences of persistently elevated body cholesterol include cholesterol gallstones, liver dysfunction, cholesterol crystal emboli, and dermatological abnormalities (e.g., xanthomas). In the case of vascular disease, the persistently high levels of circulating cholesterol-rich lipoproteins lead gradually but inexorably to their accrual in the subendothelial space of certain segments of midsize and small arteries (38) (Figure 5). This is "foreign" material, and a tenet of human physiology that is critical for survival through the reproductive years holds that foreign material must be attacked. Thus, macrophages, $\mathrm{T}$ cells, and their inflammatory cytokines enter the affected areas of the arterial wall in response to excess cholesterol-rich lipoproteins, but what they encounter is no ordinary bacteria or virus. Rather, cellular reactions to cholesterol, products of cholesterol oxidation, and other lipids that are packaged with cholesterol-carrying lipoproteins (e.g., unsaturated fatty acids) lead to a series of responses that promote the atherogenic response (38-41). Cholesterol accumulates in atherosclerotic lesions in large amounts in cells and in the extracellular space, both in esterified and in unesteri- 
fied (or "free") forms (Figure 5). Free cholesterol and certain oxidized forms of cholesterol are particularly toxic to lesional cells, and death of lesional macrophages exposed to excess free cholesterol or oxysterols is likely an important cause of lesional necrosis (ref. 37; see also Perspectives by Tabas [42] and by Björkhem [24], this series). The end result is a festering inflammatory "abscess," which all too often leads to destruction of the arterial wall, acute thrombotic vascular occlusion, and infarction of critical tissues supplied by the affected vessel $(40,41)$. Thus, a molecule essential to life is associated with the leading cause of death in the industrialized world.

\section{In this series}

The panoply of functions of cholesterol, its metabolites, and its immediate biosynthetic precursors prohibits complete coverage of all of these areas. Rather, the goal of this Perspective series is to provide a taste of interesting topics related to both the physiological and the pathophysiological roles of cholesterol. The function of cholesterol in biological membranes will be covered by several articles. Simons and Ehehalt (11) will review the role of cholesterol-mediated membrane rafts in intracellular signaling, and Maxfield and Wüstner (19) will cover various aspects related to normal and perturbed trafficking of cholesterol among cellular membranes. Travis and Kopf (14) will examine a more focused area related to cholesterol and membrane function, namely, the fascinating role of acrosomal membrane cholesterol efflux in sperm capacitation. Jefcoate (20) will deal with how cholesterol is transported to the inner mitochondrial membrane, which is critical for the conversion of cholesterol to steroid hormones. Mitochondria can also convert cholesterol into oxysterols, whose cellular and physiological functions will be reviewed by Björkhem (24). As discussed above, certain proteins become adducted with cholesterol, and Jeong and McMahon (16) will discuss how cholesterol modification affects the activity of Sonic hedgehog as a morphogen. One demonstration of the critical role played by cholesterol in normal physiology is the devastating effects of genetic mutations in sterol biosynthesis, a topic covered by Porter (18). Finally, the series includes two articles examining various aspects of the cholesterol-loaded macrophage, which is a critical cellular component of atherosclerotic lesions. Tall and colleagues (27) will review pathways of cholesterol efflux from cholesterolloaded macrophages, a key protective process that undoubtedly has important therapeutic implications. Tabas (42) will examine what happens when this and other protective functions fail, as they all too often do in atherosclerosis, by reviewing pathways by which the accumulation of unesterified cholesterol in macrophages leads to the death of these cells.

\section{Acknowledgments}

I gratefully acknowledge the comments and suggestions of Donald M. Small, Dennis Vance, Jean Vance, Thomas Haines, and John Ashkenas.
1. Bloch, K.E. 1979. Speculations on the evolution of sterol structure and function. CRC Crit. Rev. Biochem. 7:1-5.

2. Cavalier-Smith, T. 2002. The neomuran origin of archaebacteria, the negibacterial root of the universal tree and bacterial megaclassification. Int. J. Syst. Evol. Microbiol. 52:7-76.

3. Ranadive, G.N., and Lala, A.K. 1987. Sterol-phospholipid interaction in model membranes: role of C5-C6 double bond in cholesterol. Biochemistry. 26:2426-2431.

4. Haines, T.H. 2001. Do sterols reduce proton and sodium leaks through lipid bilayers? Prog. Lipid Res. 40:299-324.

5. Esfahani, M., Scerbo, L., and Devlin, T.M. 1984. A requirement for cholesterol and its structural features for a human macrophage-like cell line. J. Cell. Biochem. 25:87-97.

6. Vance, D.E., and Van den Bosch, H. 2000. Cholesterol in the year 2000. Biochim. Biophys. Acta. 1529:1-8.

7. Yeagle, P.L. 1985. Cholesterol and the cell membrane. Biochim. Biophys. Acta. 822:267-287.

8. Miao, L., et al. 2002. From lanosterol to cholesterol: structural evolution and differential effects on lipid bilayers. Biophys. J. 82:1429-1444.

9. Awad, A.B., and Fink, C.S. 2000. Phytosterols as anticancer dietary components: evidence and mechanism of action. J. Nutr. 130:2127-2130.

10. Berge, K.E., et al. 2000. Accumulation of dietary cholesterol in sitosterolemia caused by mutations in adjacent $\mathrm{ABC}$ transporters. Science. 290: $1771-1775$.

11. Simons, K., and Ehehalt, R. 2002. Cholesterol, lipid rafts, and disease. J. Clin. Invest. In press.

12. Prior, I.A., and Hancock, J.F. 2001. Compartmentalization of Ras proteins. J. Cell Sci. 114:1603-1608.

13. Simons, M., Keller, P., Dichgans, J., and Schulz, J.B. 2001. Cholesterol and Alzheimer's disease: is there a link? Neurology. 57:1089-1093.

14. Travis, A.J., and Kopf, G.S. 2002. The role of cholesterol efflux in regulating the fertilization potential of mammalian spermatozoa. J. Clin. Invest. In press.

15. Mauch, D.H., et al. 2001. CNS synaptogenesis promoted by gliaderived cholesterol. Science. 294:1354-1357.

16. Jeong, J., and McMahon, A.P. 2002. Cholesterol modification of Hedgehog family proteins. J. Clin. Invest. In press.

17. Subtil, A., et al. 1999. Acute cholesterol depletion inhibits clathrincoated pit budding. Proc. Natl. Acad. Sci. USA. 96:6775-6780.

18. Porter, F.D. 2002. Malformation syndromes due to inborn errors of cholesterol synthesis. J. Clin. Invest. In press.

19. Maxfield, F.R., and Wüstner, D. 2002. Intracellular cholesterol transport. J. Clin. Invest. In press.

20. Jefcoate, C. 2002. High-flux mitochondrial cholesterol trafficking, a specialized function of the adrenal cortex. J. Clin. Invest. In press.

21. Horton, J.D., Goldstein, J.L., and Brown, M.S. 2002. SREBPs: activators of the complete program of cholesterol and fatty acid synthesis in the liver. J. Clin. Invest. 109:1125-1131. doi:10.1172/JCI200215593.

22. Dobrosotskaya, I.Y., Seegmiller, A.C., Brown, M.S., Goldstein, J.L., and Rawson, R.B. 2002. Regulation of SREBP processing and membrane lipid production by phospholipids in Drosophila. Science. 296:879-883.

23. Russell, D.W., and Setchell, K.D. 1992. Bile acid biosynthesis. Biochemistry. 31:4737-4749.

24. Björkhem, I. 2002. Do oxysterols control cholesterol homeostasis? J. Clin. Invest. In press.

25. Rudel, L., Deckelman, C., Wilson, M., Scobey, M., and Anderson, R. 1994. Dietary cholesterol and downregulation of cholesterol 7 alphahydroxylase and cholesterol absorption in African green monkeys. J. Clin. Invest. 93:2463-2472.

26. Li, W., et al. 2002. Bile acid secreted by male sea lamprey that acts as a sex pheromone. Science. 296:138-141.

27. Tall, A.R., Costet, P., and Wang, N. 2002. Regulation and mechanisms of macrophage cholesterol efflux. J. Clin. Invest. In press.

28. Peet, D.J., Janowski, B.A., and Mangelsdorf, D.J. 1998. The LXRs: a new class of oxysterol receptors. Curr. Opin. Genet. Dev. 8:571-575.

29. Bondy, P.K. 1985. Disorders of the adrenal cortex. In Textbook of endocrinology. J.D. Wilson and D.W. Foster, editors. W.B. Saunders Co. Philadelphia, Pennsylvania, USA. 816-890.

30. Gilbert, L.I., Rybczynski, R., and Warren, J.T. 2002. Control and biochemical nature of the ecdysteroidogenic pathway. Annu. Rev. Entomol. 47:883-916.

31. Kousteni, S., et al. 2001. Nongenotropic, sex-nonspecific signaling through the estrogen or androgen receptors: dissociation from transcriptional activity. Cell. 104:719-730.

32. Stoffel-Wagner, B. 2001. Neurosteroid metabolism in the human brain. Eur. J. Endocrinol. 145:669-679.

33. Aurbach, G.D., Marx, S.J., and Spiegel, A.M. 1985. Parathyroid hormone, calcitonin, and calciferols. In Textbook of endocrinology. J.D. Wilson and D.W. Foster, editors. W.B. Saunders Co. Philadelphia, 
Pennsylvania, USA. 1137-1217.

34. Byskov, A.G., Andersen, C.Y., Leonardsen, L., and Baltsen, M. 1999. Meiosis activating sterols (MAS) and fertility in mammals and man. J. Exp. Zool. 285:237-242

35. Macko, S.A., Lubec, G., Teschler-Nicola, M., Andrusevich, V., and Engel, M.H. 1999. The Ice Man's diet as reflected by the stable nitrogen and carbon isotopic composition of his hair. FASEB J. 13:559-562.

36. Dietschy, J.M., Turley, S.D., and Spady, D.K. 1993. Role of the liver in the maintenance of cholesterol and low density lipoprotein homeostasis in different animal species, including humans. J. Lipid Res. 34:1637-1659.

37. Tabas, I. 1997. Free cholesterol-induced cytotoxicity. A possible contributing factor to macrophage foam cell necrosis in advanced ather- osclerotic lesions. Trends Cardiovasc. Med. 7:256-263.

38. Williams, K.J., and Tabas, I. 1995. The response-to-retention hypothesis of early atherogenesis. Arterioscler. Thromb. Vasc. Biol. 15:551-561. 39. Ross, R. 1999. Atherosclerosis: an inflammatory disease. N. Engl. J. Med. 340:115-126.

40. Lusis, A.J. 2000. Atherosclerosis. Nature. 407:233-241.

41. Glass, C.K., and Witztum, J.L. 2001. Atherosclerosis. the road ahead. Cell. 104:503-516.

42. Tabas, I. 2002. Consequences of cellular cholesterol accumulation: basic concepts and physiological implications. J. Clin. Invest. In press.

43. Gurr, M.I., and Harwood, J.L. 1991. Metabolism of structural lipids. In Lipid biochemistry. M.I. Gurr and J.L. Harwood, editors. Chapman \& Hall. New York, New York, USA. 297-337. 